\title{
Scaling Laws for Partially Developed Turbulence
}

\author{
Abigail Hsu*, Ryan Kaufman and James Glimm \\ Department of Applied Mathematics and Statistics, Stony Brook University, Stony Brook, NY, United States
}

We formulate multifractal models for velocity differences and gradients which describe the full range of length scales in turbulent flow, namely: laminar, dissipation, inertial, and stirring ranges. The models subsume existing models of inertial range turbulence. In the localized ranges of length scales in which the turbulence is only partially developed, we propose multifractal scaling laws with scaling exponents modified from their inertial range values. In local regions, even within a fully developed turbulent flow, the turbulence is not isotropic nor scale invariant due to the influence of larger turbulent structures (or their absence). For this reason, turbulence that is not fully developed is an important issue which inertial range study can not address. In the ranges of partially developed turbulence, the flow can be far from universal, so that standard inertial range turbulence scaling models become inapplicable. The model proposed here serves as a replacement. Details of the fitting of the parameters for the $\tau_{p}$ and $\zeta_{p}$ models in the dissipation range are discussed. Some of the behavior of $\zeta_{p}$ for larger $p$ is unexplained. The theories are verified by comparing to high resolution simulation data.

OPEN ACCESS

Edited by:

Snezhana I. Abarzhi,

University of Western Australia,

Australia

Reviewed by:

Lawrence Forbes,

University of Tasmania, Australia

Prabir Daripa,

Texas A\&M University, United States

*Correspondence:

Abigail Hsu

abigail.hsu2009@gmail.com

Specialty section:

This article was submitted to

Dynamical Systems,

a section of the journal

Frontiers in Applied Mathematics and

Statistics

Received: 10 November 2021 Accepted: 27 December 2021

Published: 20 January 2022

Citation:

Hsu A, Kaufman R and Glimm J (2022) Scaling Laws for Partially

Developed Turbulence.

Front. Appl. Math. Stat. 7:812330.

doi: 10.3389/fams.2021.812330
Keywords: turbulence, multifractals, scaling law, structure functions, intermittency

\section{INTRODUCTION}

We develop a conceptual framework for turbulent scaling laws across length scales extending beyond the inertial range. Classically, fully developed turbulence is defined as occurring on length scales in which energy transfer is dominated by inertial forces. Partially developed turbulence is defined as turbulent (i.e., non-laminar) ranges with significant dissipation or significant stirring. Thus, length scales with significant dissipation and stirring forces are included within partially developed turbulence. We propose new models with supporting verification which subsume and extend inertial and non-inertial range models of others. From our models and data analysis, we explain phenomena not previously observed and phenomena previously observed but not explained.

The inertial range is defined as an intermediate range of length scales, $l$, which are far from the Kolmogorov scale $\eta$ and integral scale $L$, i.e., $\eta \ll l \ll L$. The dissipation range is the range of length scales between the laminar range and the inertial range. The stirring range can be at any length scale, but in common experiments, observations and simulations, stirring is at large scales. The influence of stirring decreases (in a controlled and scaling measurable manner) outside for scales smaller than those actually stirred. This line of investigation, while observed by the authors, has not been developed systematically in the present paper and could be addressed in the future.

Structure functions give a precise meaning to clustering of bursts of turbulent intensity and compound clustering (i.e., clustering of clusters) etc. They measure the dependence of these compound clustering rates on the length scale set by the observational size of the cluster. Two families of structure functions are studied here: one characterizes the powers of the energy dissipation rate, $\epsilon$, and the other characterizes powers of the velocity difference, $\delta u$. 
The turbulent dissipation rate can be derived from the turbulent velocity gradients as:

$$
\epsilon(\vec{x}, t)=\frac{v}{2}\left(\frac{\partial u_{i}}{\partial x_{j}}+\frac{\partial u_{j}}{\partial x_{i}}\right)^{2}
$$

where $v$ is the kinematic viscosity of the fluid and $u$ is the fluctuating velocity, and the summation convention is applied to repeated indices represented the three Cartesian directions by following the definition by [1].

We define the coarse grained, local average of the dissipation rate $\epsilon_{l}$ as,

$$
\epsilon_{l}(\vec{x})=\frac{1}{\left|V_{l}\right|} \iiint_{V_{l}(\vec{x})} \epsilon(\vec{y}, t) \mathrm{d} \vec{y}
$$

where $V_{l}(\vec{x})$ is a volume with a diameter $l$ centered around $\vec{x}$ in $3 \mathrm{D}$. The coarse grained averaging means that $\epsilon_{l}$ reflects properties occurring on the length scale $l$.

The structure functions of $\delta_{l} u$ and $\epsilon_{l}$ satisfy asymptotic scaling relations as a power of the length scale $l$. The structure functions and the associated scaling exponents $\tau_{p}$ and $\zeta_{p}$ are given by the expectation relations,

$$
\left\langle\left(\epsilon_{l}\right)^{p}\right\rangle \sim l^{\tau_{p}} \quad \text { and } \quad\left\langle\left|\delta_{l} u\right|^{p}\right\rangle \sim l^{\zeta_{p}}
$$

The length scale dependent scaling exponents $\tau_{p}$ and $\zeta_{p}$ are obtained from logarithmic local slopes, i.e.,

$$
\tau_{p}=\frac{d\left(\ln \left\langle\epsilon_{l}^{p}\right\rangle\right)}{d(\ln l)} \quad \text { and } \quad \zeta_{p}=\frac{d\left(\ln \left\langle\left|\delta_{l} u\right|^{p}\right\rangle\right)}{d(\ln l)}
$$

as suggested by [2].

The main thrust of the theory is that turbulent scaling laws remain in effect across all length scale ranges, but with modified length dependent scaling exponents.

A key step in the parameterization of the introduced scaling models is that $\tau_{p}$ and $\zeta_{p}$ in Equation (4) are linear in $\ln l$ in the dissipation range. Hence, the slopes of $\tau_{p}$ and $\zeta_{p}$ are constructed,

$$
T_{p}=\frac{d\left(\tau_{p}\right)}{d(\ln l)} \quad \text { and } \quad Z_{p}=\frac{d\left(\zeta_{p}\right)}{d(\ln l)}
$$

which are modeled across all length scales as piecewise constant.

The piecewise constant values of $T_{p}$ and $Z_{p}$ appear to be a new discovery. These piecewise constant values over the 4 length scale ranges are described as follows:

$$
\begin{aligned}
& T_{p}= \begin{cases}0, & \text { laminar range (LR) } \\
T_{p}^{d r}, & \text { dissipation range (DR) } \\
0, & \text { inertial range (IR) } \\
T_{p}^{s r}, & \text { stirring range (SR) }\end{cases} \\
& Z_{p}= \begin{cases}0, & \text { laminar range (LR) } \\
Z_{p}^{d r}, & \text { dissipation range (DR) } \\
0, & \text { inertial range (IR) } \\
Z_{p}^{s r}, & \text { stirring range (SR) }\end{cases}
\end{aligned}
$$

In the inertial range, we take $T_{p}=0$. We do not observe $T_{p}=0$ from our data in this range, but include this to be consistent with the classical model by [3], denoted SL, in lieu of a more comprehensive theory.

$T_{p}^{d r}$ and $Z_{p}^{d r}$ are constant in the dissipation range, and $T_{p}^{s r}$ and $Z_{p}^{s r}$ are constant in the stirring range for the problems we study. These $T_{p}$ and $Z_{p}$ values are verified in the JHTDB and the $T_{p}$ values in the UMA data shown in section 4.1 addressing the laminar region where the eddy length scale is smaller than the Kolmogorov microscale $\eta$. In addition, we observe that $T_{p}$ is linear in $p$, and $Z_{p}$ is given by an explicit $p$ dependent formula in the dissipation range.

In the dissipation range, the logarithmic dissipation rate is proportional to $\ln (l)$. In contrast, the dissipation defined in the Navier-Stokes equation itself occurs in the laminar range at a rate proportional to the length scale $l$. For $p=2$, the laminar energy dissipation rate $\epsilon$ includes the classical viscosity $v$ within its definition. The increase of both $\tau_{p}$ and $\zeta_{p}$ for all $p$ in a limit as $l$ approaches $\eta$ from above leads to negative constant values of $T_{p}$ and $Z_{p}$ in their respective dissipation ranges.

The key property of constant slopes $T_{p}$ and $Z_{p}$ is also satisfied in the stirring range and observed in the JHTDB data. In principle, stirring forces can be added in an arbitrary manner at any length scale. Parameterization of the stirring range is not addressed in this paper.

We provide a brief literature review in section 2. The numerical verification data from JHTDB and UMA are described in section 3 as well as the methods used for data analysis. The scaling law results, which are the technical core of the paper, are presented in sections 4,6 . The extended and refined scaling analytical methods are discussed in section 5. A comment on the asymptotics of the viscous limit can be found in section 7 . Conclusions are summarized in section 8 .

\section{INERTIAL RANGE PRIOR RESULTS}

Kolmogorov [4], denoted K41, postulated universal laws to govern the statistics on all such length scales in the inertial range in which the flow is statistically self similar. Dimensional analysis in $\mathrm{K} 41$, based on the self similarity hypothesis, led to the $-5 / 3$ scaling law. However, because the energy dissipation for turbulent flows is intermittent, this model has been refined in various ways over the years to yield a multifractal scaling law. Summarized in [5], the K41 exponent $\zeta_{p}$ is modified to capture the compound clustering of turbulent structure using a multifractal analysis. Kolmogorov [6] refined his similarity hypothesis, denoted K62, added the influence of the large flow structure and included the influence of the intermittency. The refined similarity hypothesis in [6] for the classical inertial range links the scaling exponent $\zeta_{p}$ of the longitudinal velocity structure and the scaling exponent $\tau_{p}$ of the energy dissipation rate as

$$
\left\langle\left|\delta_{l} u\right|^{p}\right\rangle \sim\left\langle\epsilon_{l}^{p / 3}\right\rangle l^{p / 3},
$$

where $\left\langle\epsilon_{l}^{p / 3}\right\rangle \sim l^{\tau_{p / 3}}$, or equivalently

$$
\zeta_{p}=\frac{p}{3}+\tau_{\frac{p}{3}}
$$


The log-normal model from SL defines a theoretical model for the PDF of the coarse-grained energy dissipation in the inertial range. SL studied the quantity $\epsilon_{l}^{(p)}$ defined as the ratio:

$$
\epsilon_{l}^{(p)}=\frac{\left\langle\epsilon_{l}^{p+1}\right\rangle}{\left\langle\epsilon_{l}^{p}\right\rangle} .
$$

where $p$ can be any non-negative integer. The $\epsilon_{l}^{(0)}$ and $\epsilon_{l}^{(\infty)}$ are related to the mean fluctuation structure $\bar{\epsilon}$ and the filamentary structure. The scaling law for $p \rightarrow \infty$ is $\epsilon_{l}^{(\infty)} \sim l^{-2 / 3}$. As $p \rightarrow \infty$, the definition of $\tau_{p}$ stating that,

$$
\tau_{p+1}-\tau_{p} \longrightarrow-\frac{2}{3}
$$

or $\tau_{p}=-\frac{2}{3} \cdot p+C$. The codimension $C$ is evaluated as $C=3-1=2$ based on the assumption that the elementary filamentary structures have dimension 1 . The expectation $\left\langle\epsilon_{l}^{p}\right\rangle$ has an $l$ dependence which is not a pure exponential, but a mixture of exponentials, i.e., the scaling exponents for $\left\langle\epsilon_{l}^{p}\right\rangle$ are defined as a weighted average of exponentials.

From the assumption of the interaction between structures of different order, SL proposed the following relation between structures of adjacent order:

$$
\epsilon_{l}^{(p+1)}=A_{p} \epsilon_{l}(p)^{\beta} \epsilon_{l}^{(\infty)^{1-\beta}}
$$

Based on Equations $(9,11)$, SL derives a two step recursion for $\tau_{p}$. This recursion relation implies that $\tau_{p}=-\frac{2}{3} \cdot p+2+f(p)$, where $f(\infty)=0$ is assumed. The equation for $f(p)$ has the solution $f(p)=\alpha \beta^{p}$ and with the boundary conditions $\tau_{0}=\tau_{1}=0$, the solution becomes

$$
\tau^{S L}(p)=-\frac{2}{3} \cdot p+2\left[1-\left(\frac{2}{3}\right)^{p}\right]
$$

Substituting Equation (12) into the relation shown in (8) yields

$$
\zeta_{p}=\frac{p}{9}+2\left[1-\left(\frac{2}{3}\right)^{\frac{p}{3}}\right]
$$

Novikov [7] suggested that the $-2 / 3$ on the RHS of Equation (10) should be replaced by -1 based on the theory of infinitely divisible distributions applied to the scaling of the locally averaged energy dissipation rate $\epsilon_{l}$. Chen and Cao [8], denoted CC, accepted Novikov's suggestions and derived the formula:

$$
\tau^{C C}(p)=-p+\left[\left(1+\tau_{2}\right)^{p}-1\right] / \tau_{2},
$$

which uses the classical value $\tau_{2} \approx-0.22$, which is derived from simulations, observations, experiments, and theory (SL) all in approximate agreement.

Kolmogorov proposed that $\zeta_{3}=1$ for incompressible, isotropic and homogeneous turbulence. Frick et al. [9] showed that in the case of non-homogeneous shell models with $\zeta_{3} \neq$
1 , the scaling of velocity structure functions in incompressible turbulence from SL still holds as $\zeta_{p} / \zeta_{3}=p / 9+2\left[1-(2 / 3)^{\frac{p}{3}}\right]$.

Boldyrev et al. [10] predicted a new scaling law for the scaling exponent $\zeta_{p}$ of velocity structure functions as

$$
\zeta_{p} / \zeta_{3}=p / 9+1-(1 / 3)^{\frac{p}{3}}
$$

in supersonic turbulence for star formation based on a Kolmogorov-Burgers model. The same behavior is observed by [11] in incompressible MHD.

The SL model has generated a considerable interest in the hierarchical nature of turbulence. Experiments and simulations have been conducted to evaluate the velocity and energy dissipation structures. Chavarria et al. [12-14] demonstrated experimental variables for the hierarchical structure assumption for the function $\zeta_{p}$ in Equation (13). Experimental studies on a turbulent pipe flow and a turbulent mixing layer by [15] verified the SL hierarchical symmetry. Cao et al. [16] showed agreement between the SL scaling exponents and high-resolution direct numerical simulations (DNS) of 3D Navier-Stokes turbulence.

Chevillard et al. [17] clearly frames the problem we address as the major theme of our paper. Their model and ours study the $\zeta_{p}$ and $\tau_{p}$ exponents in the turbulent dissipative regime. Their predictions are limited to the structure of order 4 or less. The validation results are complicated by differences in the experimental data, but each of their plots shows agreement with some of the data. Briefly, our results go beyond this work in several respects. We model the scaling exponent $\zeta_{p}$ and $\tau_{p}$ scaling asymptotics, each with two parameters, giving both the scaling exponent and the multiplying coefficient. We set these (4 in total, $\mathrm{p}$ dependent) parameters for values up to $p=10$ on $\zeta_{p}$ and up to $p=30$ on $\tau_{p}$, with consistent validation against two independent sets of simulation data.

We relate our modeling to a variety of other and more recent studies of the setting of parameters for the She Levegue model. Traditionally, stirring is introduced at the large length scales and becomes less significant at the smaller ones as has been studied [18-20] previously. We also observed the same effect, and in our study of the JHTDB, found scaling laws similar to that reported in the dissipative regime, as is suggested in the drawing of Figures 2-4.

\section{METHODS}

\subsection{JHTDB Data}

We analyze the DNS data of the forced isotropic turbulence simulation from the Johns Hopkins Turbulence Database (JHTDB) performed by [21] and [22]. The simulated flow has an integral scale Reynolds number $R e=23,298$ and a Taylor scale Reynolds number $R e_{\lambda}=433$. The JHTDB data are generated by direct numerical simulation of forced isotropic turbulence in a cubic domain with length $L=2 \pi$ and periodic boundary conditions in each direction. The simulation has a resolution of $1024^{3}$ of cells. Energy is injected to maintain a constant value for the total energy. The JHTDB data are collected after the 
simulation has reached a statistical stationary state. The data are posted on the website http://turbulence.pha.jhu.edu The JHTDB data focus on analysis of the inertial range. Because of this emphasis, its coverage of the dissipation and stirring ranges is limited. The ratio of the Kolmogorov length scale to the computational grid space is $\eta / \Delta x=0.46$. Thus, the JHTDB data do not fully resolve the Kolmogorov length scale $\eta$. With these data, we confirm many aspects of our scaling law model. We anticipate the need for additional simulation data such as the $4096^{3}$ cell data from the JHTDB in further analysis of laminar and dissipation ranges.

\subsection{JHTDB Data Analysis}

The velocity differences have a tensorial dependence on the velocity component directions and the differencing directions. The longitudinal direction is more convenient for experiments, and many experimental prior studies focused on the longitudinal velocity increment based on Taylor's hypothesis. Details are described by [23]. We define the longitudinal velocity increment as $\delta_{l} u(x, y, z, t)=u(x+l, y, z, t)-u(x, y, z, t)$, where $u$ is the $x$ component of velocity.

The coarse-graining length scale, $l$, in the structure function definition, Equation (2) is implemented by a $3 \mathrm{D}$ average over distances of a scale $l$, and $\epsilon$ is given at discrete locations in space. From previous literature, the averaging volume is taken to be a sphere or a cube. The definition of $l$ as radius or diameter is not consistent in the literature. In this paper, we distinguish between spherical and cubic average with a diameter $l$. The dominant effects on the averaging element, sphere or cube, come from the most extreme points on the elements, i.e., the boundary of the sphere or corners of the cube, and always with these extreme points at distance $l / 2$ from the center.

In Figure 1, we observe an approximate but not exact agreement between the cubic and spherical averages. The cubic average, producing a consistent but less noisy range, is used for the local average of the energy dissipation rate in this paper.
A further observation is that $\tau_{2}$ in the inertial range is not consistent with prevailing theory i.e., $\tau_{2}$ is not constant while $\zeta_{2}$ is constant in this range. Figure 1 shows the theoretical value for the inertial range $\tau_{2}^{S L}$ as a horizontal line. The local slope measurements are points connected by line segments. While an average across a large range is consistent with SL, no universal constant local slope is found that is consistent with $\tau_{2}^{S L}$. For this reason, we regard the $\tau_{p}$ theory from SL more exactly as a theory for $\zeta_{p}$ rather than a theory for $\tau_{p}$.

\subsection{UMA Data Analysis}

The UMA data from [24] are based on a DNS of isotropic homogeneous turbulence from the University of Massachusetts Amherst (UMA). This simulation uses a third-order AdamsBashforth and pseudo-spectral method. The simulation uses a periodic cube with edge length $2 \pi$ and a $2048^{3}$ numerical grid. The simulation parameters are $\eta / \Delta x=3.04$ and $\eta k_{\max }=6.4$. The flow has an integral scale Reynolds number $R e=3,426$ and a Taylor scale Reynolds number $R e_{\lambda}=151$. The inertial range turbulence is fully developed and the data also describe with a complete dissipation range with length scales smaller than the Kolmogorov length scale. There are sufficient data to provide verification in the laminar range. We digitized the local slopes $-\tau_{p}$ from the published UMA data for $p=2,3,4$ by [24].

The UMA locally averaged dissipation rate $\epsilon_{l}$ data are defined as a spherical average with a diameter $l$. An interpolation consistent with the numerical method is used to solve the governing equations and allows the elimination of the noise.

As noted in Figure 1, the spherical average leads to a higher $-\tau_{p}$ value than the cubic average. A vertical shift of 0.04 for $-\tau_{2}$ of the cubic averaged JHTDB data is needed to reach the spherical averaged UMA maximum for comparison. In addition, a horizontal shift of the normalized length scale $l / \eta$ is needed to compensate for the JHTDB under resolution of the Kolmogorov scale.

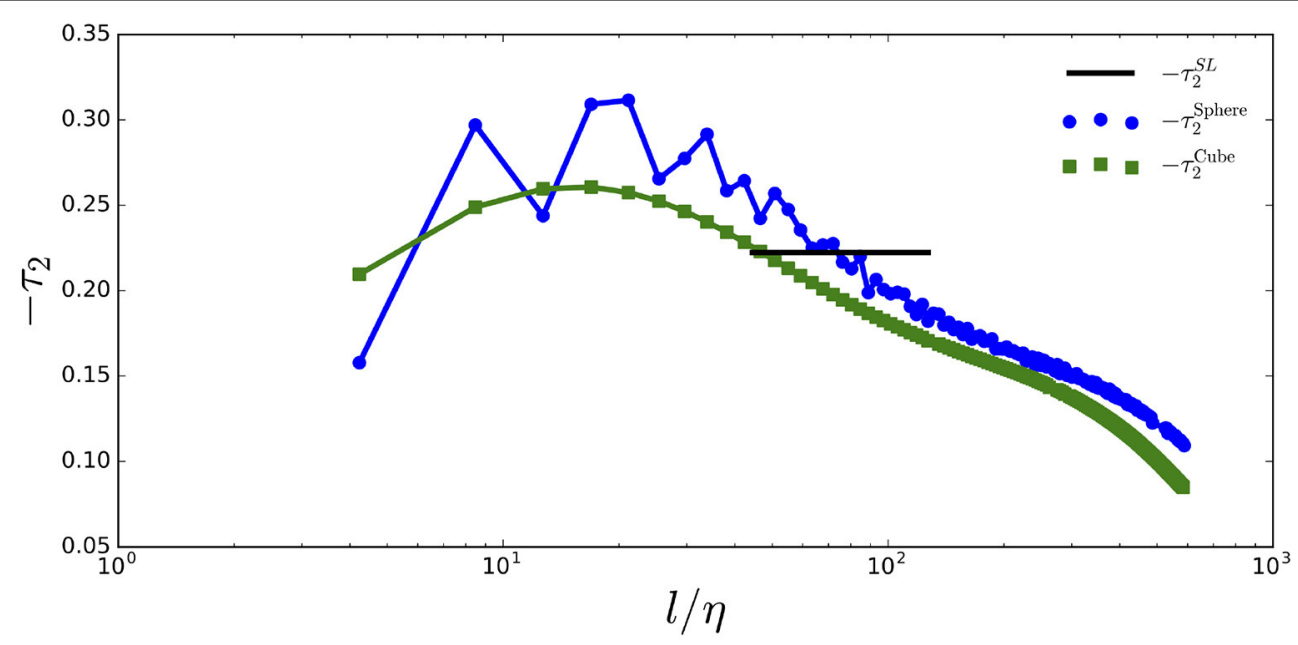

FIGURE 1 | Comparison of the cubic to the spherical average with a diameter / for $\epsilon_{l}$ in the computation of $-\tau_{2}$. The horizontal solid line is the constant local exponent $-\tau_{2}^{S L}$ value from SL and is located at the inertial range that is determined by $\zeta_{2}$ from JHTDB. 


\subsection{Schematic Model Formulation}

Figure 2 summarizes our major ideas. The laminar, dissipation, inertial and stirring ranges are labeled. This figure displays the slopes, $T_{p}$, as linear segments in the dissipation and stirring ranges. In Figure 1, $-\tau_{2}$ does not show a clear flat range to indicate the classical inertial range. The flat segment shown in the schematic representation is taken from the SL theory. We comment on its absence from the JHTDB and UMA data in section 4.3.

Figures 3, 4 show that as $p$ increases, so do the values for $-\tau_{p}$ and $\zeta_{p}$. Laminar flow occurs at a nearly zero and is shown as a nearly horizontal line on the semi-log plots in Figures 2-4. The linearity of $\tau_{p}$ in the dissipation range implies the equation

$$
\tau_{p}=T_{p} \ln \left(\frac{l}{\eta}\right)+b_{p}
$$

where $T_{p}$ represents the constant slope observed in $\tau_{p}$ and $b_{p}$ is the $\tau_{p}$ value at the Kolmogorov length scale $(\eta)$.

Similarly, in the dissipation range, we define

$$
\zeta_{p}=Z_{p} \cdot \ln \left(\frac{l}{\eta}\right)+a_{p},
$$

where $Z_{p}$ represents the constant slope observed in $\zeta_{p}$ and $a_{p}=$ $\zeta_{p}$ at the Kolmogorov length scale $(\eta)$.

A transition point in the schematic representation occurs at the length scale where the slope of $\tau_{p}$ or $\zeta_{p}$ changes. The transition from turbulent dissipation to laminar dissipation occurs approximately at $l=\eta$. The detailed locations of the transition are dependent on $p$ and are different for $\zeta_{p}$ and $\tau_{p}$. The transition actually occurs gradually rather than discontinuously as we see in Figure 5. However, in our modeling, $T_{p}$ and $Z_{p}$ are piecewise constant in $\ln l$ as an approximation to an exact theory. All other transitions are determined similarly.

In this modeling, $\zeta_{p}$ or $\tau_{p}$ for each $p$ defines its own inertial range. The SL model holds within the inertial range defined by $\zeta_{p}$.

\section{SCALING LAWS FOR THE ENERGY DISSIPATION RATE}

\subsection{The Laminar Range}

In the laminar region, velocity fluctuation is zero, so dissipation is no longer at a power law in the exponent and operates on a

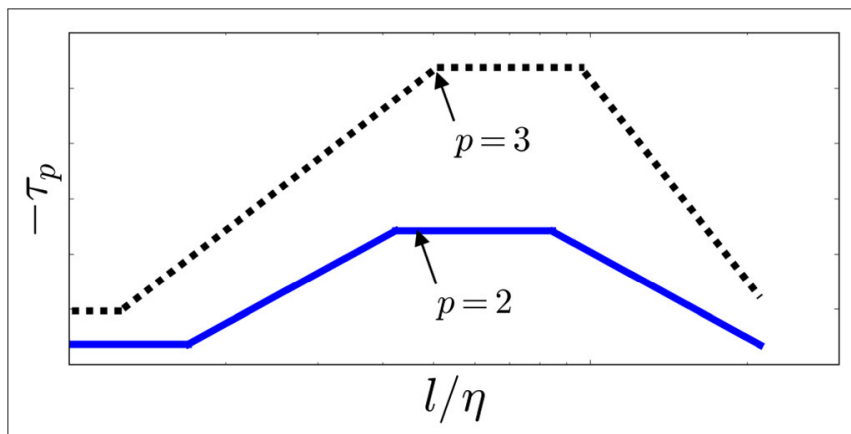

FIGURE 3 | Schematic representation of the local slope, $-\tau_{p}$ vs. log length scale with the $p$-dependence shown by the arrow.



FIGURE 4 | Schematic representation of the local slope, $\zeta_{p}$ vs. log length scale with the $p$-dependence shown by the arrow.

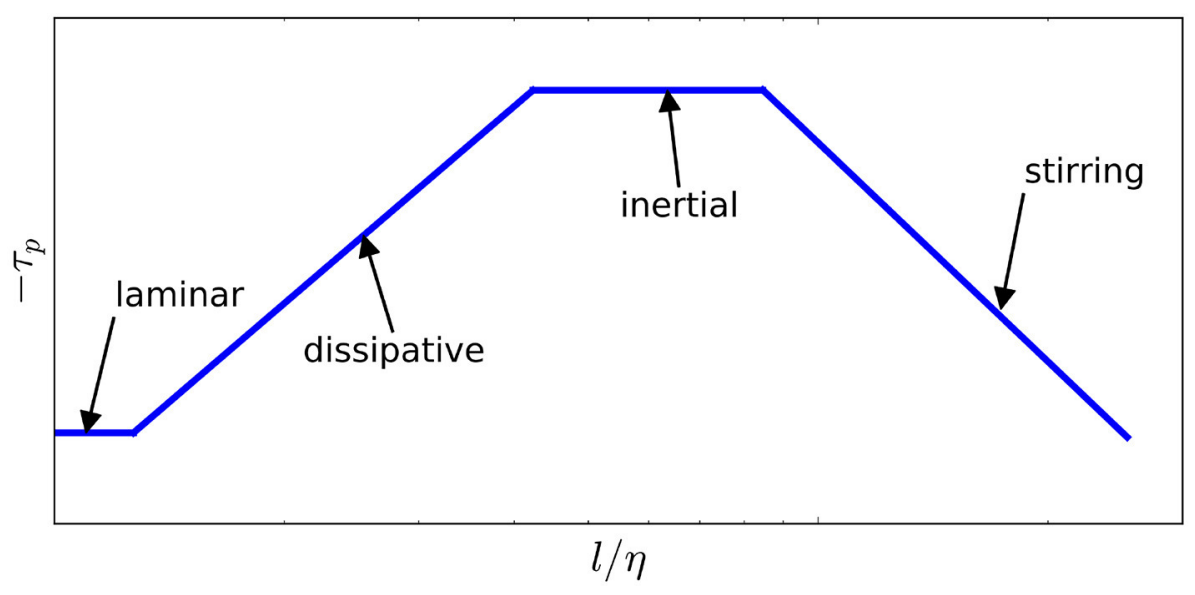

FIGURE 2 | Schematic representation of $-\tau_{p}$ Vs. $I / \eta$ on a semi-log scale. 
linear scale as normal NS small amplitude. The digitized UMA data of $-\tau_{p}$ for $p=2,3,4$ in the laminar range is shown in Figure 5. $-\tau_{2}$ and $-\tau_{3}$ are close to zero here with, perhaps, a small $p$ dependence shown in the $\tau_{4}$ data. The transition is observed to be gradual in the simulation data analyzed from an exponential point of view. Thus, we assume that the $\tau_{p}$ values are approximately zero in the laminar range in which $l / \eta<1$.

\subsection{The $p$-Dependent Linear Slopes in the Dissipation Range}

The UMA data of Figure 6 and the JHTDB of Figure 7 show the linear segment of $\tau_{p}$ in the dissipation range. With limited data in small length scales but including higher $p$ values (up to $p=10$ ), we reach the same conclusion from Figure 8 for the JHTDB data. The solid lines are modeled by the $C C_{2}$ model and will be explained in section 5 . The linear slopes are determined by least squares.

Recall that $\tau_{p}=T_{p} \ln (l / \eta)+b_{p}$. In Figure 9, we plot the $T_{p}$ for $p$ up to 30 in the dissipation range. The slopes, $T_{p}$, are linear in $p$, with a $T_{2}$ data dependence, i.e.,

$$
T_{p}=\frac{d T_{p}}{d p} \cdot p+\left(T_{2}-2 \cdot \frac{d T_{p}}{d p}\right) .
$$

We find that $d T_{p} / d p=-0.323$ from the JHTDB data.

\subsection{Full $\tau_{p}$ Parameterization}

We have developed a model that captures all of the length scales from the laminar range up to the small length scale end of inertial range for the energy dissipation rate Based on Equation (18) and

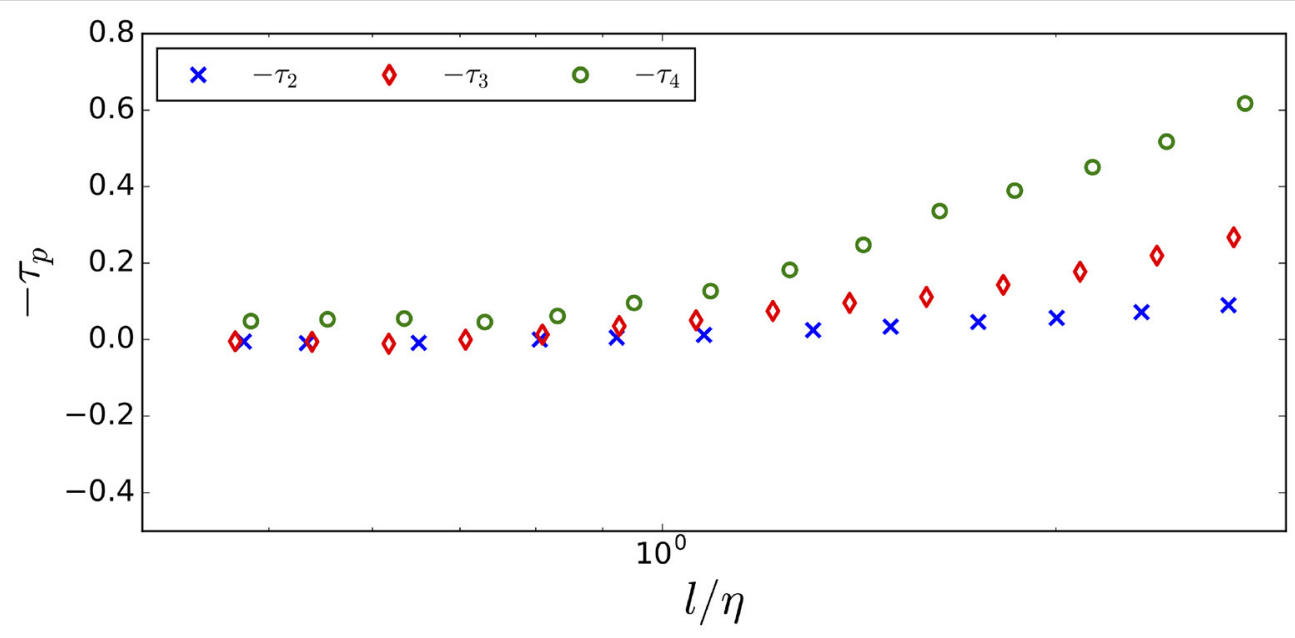

FIGURE 5 | The digitized $-\tau_{p}$ values from the UMA data enlarged for the laminar range, for $p=2,3,4$. We observe constant $-\tau_{p}$ values with weak $p$ dependence for smaller lengths.



FIGURE 6 | The digitized $-\tau_{p}$ UMA data. The horizontal solid lines are the constant local exponent $-\tau_{p}$ values from SL with the inertial range determined by $\zeta_{2}$ from JHTDB. The vertical line marks the Taylor micro scale. 


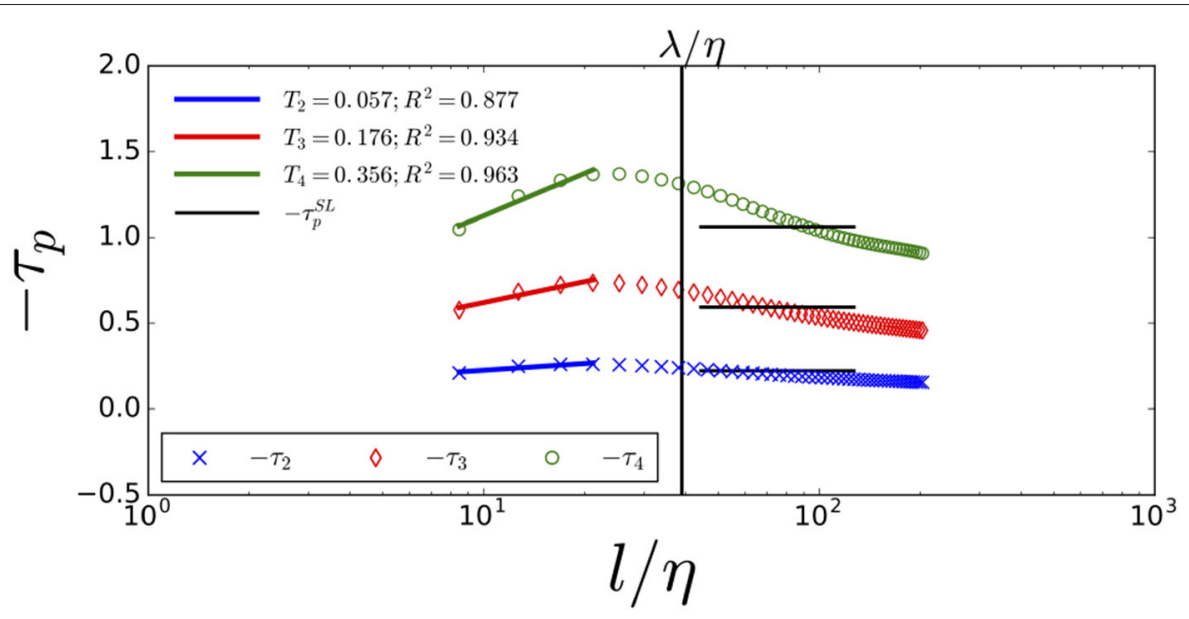

FIGURE 7 | The local slopes $-\tau_{2},-\tau_{3},-\tau_{4}$ from the JHTDB data are linear in $\ln (I / \eta)$ in the dissipative ranges, with constant slopes $T_{p}$. An $R^{2}$ value closes to 1 indicates the goodness of the fit.



FIGURE 8 | The local slope $\tau_{p}$ for all $2 \leq p \leq 10$ with $p$ increasing from the bottom to the top. The JHTDB data are shown in scatter points and the modeled $-\tau_{p}$ values are in solid lines from the $\mathrm{CC}_{2}$ model explained in section 5 .

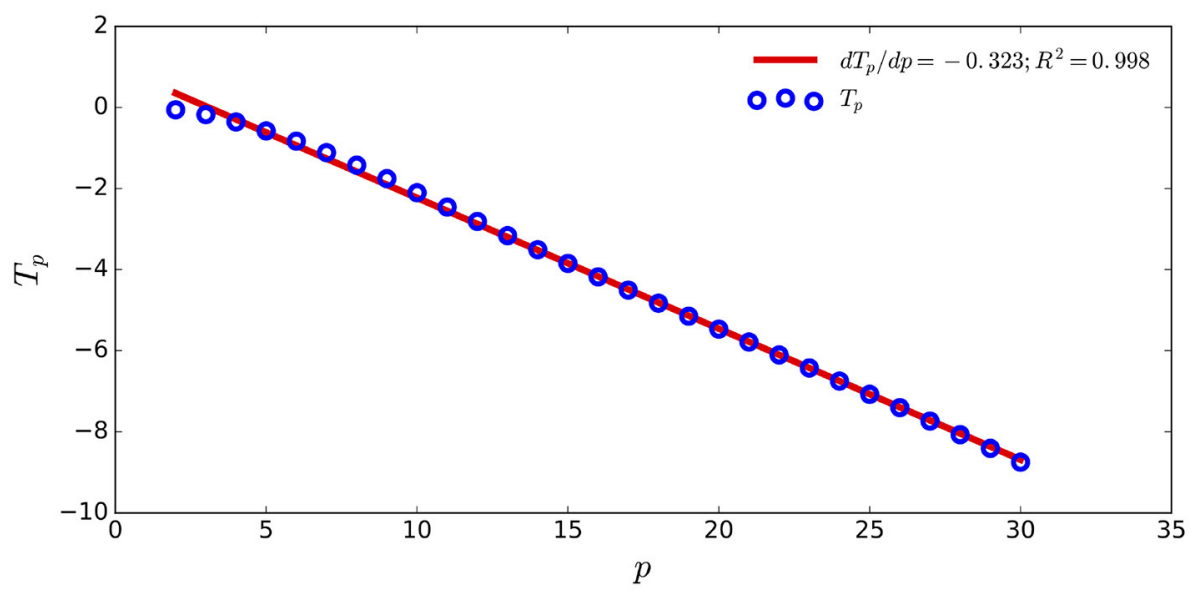

FIGURE 9 | The $T_{p}$ in the dissipative range is linear for $p$ in the range $2 \leq p \leq 30$ for the JHTDB data. 
the assumption of $\tau_{p} \approx 0$ in the laminar range. A model for $\tau_{p}$ in the dissipation range is

$$
\tau_{p}^{d r}=\left(\frac{d T_{p}}{d p} \cdot p+\left(T_{2}-2 \cdot \frac{d T_{p}}{d p}\right)\right) \cdot \ln \left(\frac{l}{\eta}\right)
$$

which can be extended for length $l$ up to the Taylor micro-scale $\lambda$.

In the JHTDB and UMA data, there is no flat inertial range observed for $\tau_{p}$ and the $\tau_{p}$ peak occurs approximately at the Taylor micro-scale $\lambda$. The peak could be an isolated point represents a transition to the stirring range. Alternatively, there maybe a small inertial range and a large transition range, in which case, it is possible that with higher Reynolds number, a true (flat) inertial range for $\tau_{p}$ may appear.

\section{THE EXTENDED AND REFINED CC MODELS FOR THE SCALING EXPONENT OF THE ENERGY DISSIPATION RATE}

The CC model defined by [8] shown in Equation (14) has a dependence on inertial constant $\tau_{2}=\tau_{2}^{S L} \approx-0.22$ value from SL. We extend the model beyond the inertial range, where a constant $\tau_{2}^{S L}$ is no longer an accurate value for length scale dependent $\tau_{2}$. To calculate $\tau_{p}$ for $p>2$, we substitute the $\tau_{2}$ data that are measured across all length scales into Equation (14). The extended model is denoted as the $C C_{2}$ model. Figure 10 shows this model for $\tau_{3}$ (solid line) in comparison to the observed $\tau_{3}$ value (data points). The same comparison for $p \leq 10$ is shown in Figure 8. The data and model values agree for small $p \leq 5$, but diverge at higher $p$ values. We propose a modification of $C_{2}$, which we denote as $C C_{q}$ model. Instead of taking a measured $\tau_{2}$, as in our extended $C C_{2}$ model, we substitute the measured $\tau_{q}$ data that is available across all measurable length scales, and solve numerically for $\widehat{\tau}_{2}$ shown as below:

$$
\tau_{q}=-q+\left[\left(1+\widehat{\tau}_{2}\right)^{q}-1\right] / \widehat{\tau}_{2} .
$$

Then, the calculated $\widehat{\tau}_{2}$ can be substituted back to Equation (14) to calculate $\tau_{p}$ for any $p$. From this point, all $\tau_{p}$ values can be modeled as a function of $p$ and the measured $\tau_{q}$ using the $C C_{q}$ model.

Figure 11 shows the linear fit $T_{2}$ of $\tau_{2}$ that is calculated from the $C C_{q}$ model using measured $\tau_{q}$ values in the dissipation range, for $q=2,3, \ldots, \infty$. This sequence of $T_{2}$ values are fitted by:

$$
T_{2}=0.229 / q+0.001
$$

for any $q$ value. $T_{2}$ decreases and converges asymptomatically to 0.001 as $q \rightarrow \infty$. Hence, the $C C_{\infty}$ model is defined as $C C_{\infty}=$ $\lim _{q \rightarrow \infty} C C_{q}$.

\section{SCALING LAWS FOR THE LONGITUDINAL VELOCITY INCREMENT STRUCTURE FUNCTIONS}

\subsection{The $p$-Dependent Linear Slopes in the Dissipation Range}

The JHTDB data for the slopes $Z_{p}$ of the longitudinal velocity increment $\left\langle\left|\delta_{l} u_{1}\right|^{p}\right\rangle$ vs. $\ln (l / \eta)$ for $p=2,3,6$ are shown in Figure 12. We see that $\zeta_{p}$ is consistent with the horizontal solid lines of $\zeta_{p}^{S L}$ values obtained from Equation (13) in the inertial range. In addition, $\zeta_{p}$ is linear in $\ln (l / \eta)$ in the dissipation range. Summary data for all $p$ up to 10 are given in Figure $\mathbf{1 3}$ with linear extrapolation from the dissipation range to the Kolmogorov scale for the JHTDB data.

Recall that $\zeta_{p}=Z_{p} \ln \left(\frac{l}{\eta}\right)+a_{p}$ in our linear model for longitudinal velocity increment in the dissipation range. This equation gives a new relation for the linear slope ratio $Z_{p} / Z_{3}$ and

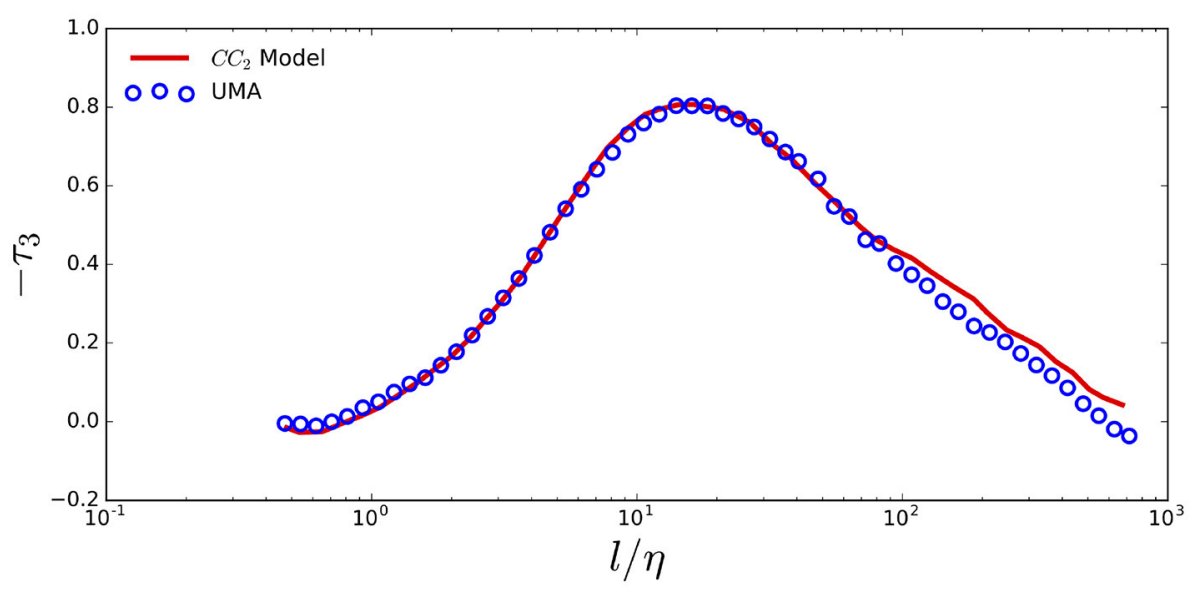

FIGURE 10 | The extended $C C_{2}$ model for $-\tau_{3}$ shows agreement with the UMA data across all measurable length scales. 

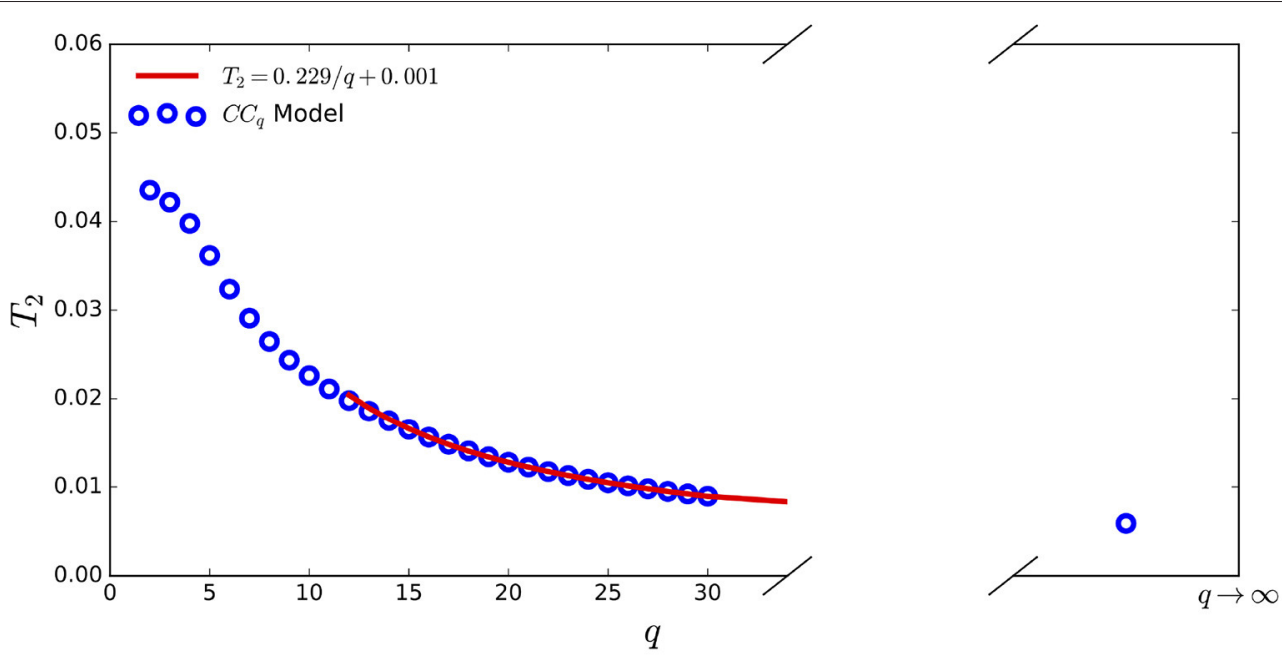

FIGURE 11 The linear slopes $T_{2}$ of $\tau_{2}$ calculated from the $C C_{q}$ model for $q$ from 2 to 30 using JHTDB is plotted vs. $q$ in the dissipative range.

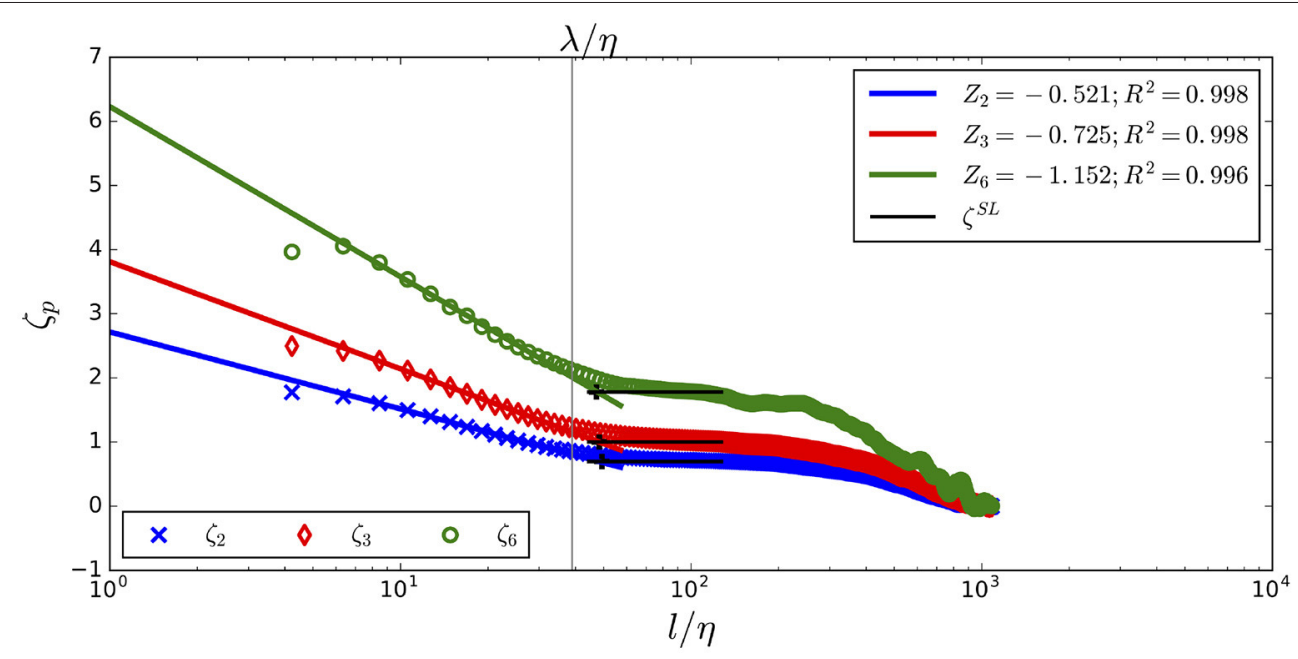

FIGURE $12 \mid \zeta_{2}, \zeta_{3}, \zeta_{6}$ are linear in the dissipative ranges with the solid line representing the linear fit of $\zeta_{p}$ in the dissipation range, extrapolated to the Kolmogorov scale for the JHTDB data. The horizontal lines are the corresponding SL model values and the vertical line marks the Taylor micro-scale. The bold "+" near the Taylor scale values $\lambda / \eta$ are the transition points from the dissipation ranges to the inertial ranges.

the ratio $a_{p} / a_{3}$ as:

$$
\begin{aligned}
\zeta_{p} / \zeta_{3} & =\frac{Z_{p} \cdot \ln \left(\frac{l}{\eta}\right)+a_{p}}{Z_{3} \cdot \ln \left(\frac{l}{\eta}\right)+a_{3}} \\
& =\frac{Z_{p}+a_{p} / \ln \left(\frac{l}{\eta}\right)}{Z_{3}+a_{3} / \ln \left(\frac{l}{\eta}\right)} .
\end{aligned}
$$

Based on the model representation (15) and on Equation (22), we develop a model for the longitudinal velocity structure in the dissipation range as:

$$
\begin{aligned}
& Z_{p} / Z_{3}=p / 9+1-(1 / 3)^{\frac{p}{3}}, \\
& a_{p} / a_{3}=p / 9+1-(1 / 3)^{\frac{p}{3}} .
\end{aligned}
$$

This law is valid for the JHTDB data up to $p=10$ as shown in Figure 14.

Given $Z_{3}, Z_{p}$ can be modeled in the dissipation range by Equation (6.1). Note $\zeta_{15}$ is not linear in $\ln (l / \eta)$ in the dissipation range. This is typical for $p>10$, so we do not include a model for $\zeta_{p}$ for larger $p$. The mismatch between the data and model for larger $p$ raises the question whether the model needs to be improved, the numerical methods are unstable for high moments, or the data need improvement. 




FIGURE 13 | The JHTDB defined $\zeta_{p}$ with $p$ increasing from 1 to 10 from the bottom to the top across all available length scales. The plot shows scatter points with solid lines representing the linear fit of $\zeta_{p}$ in the dissipation range extrapolated to the Kolmogorov scale.

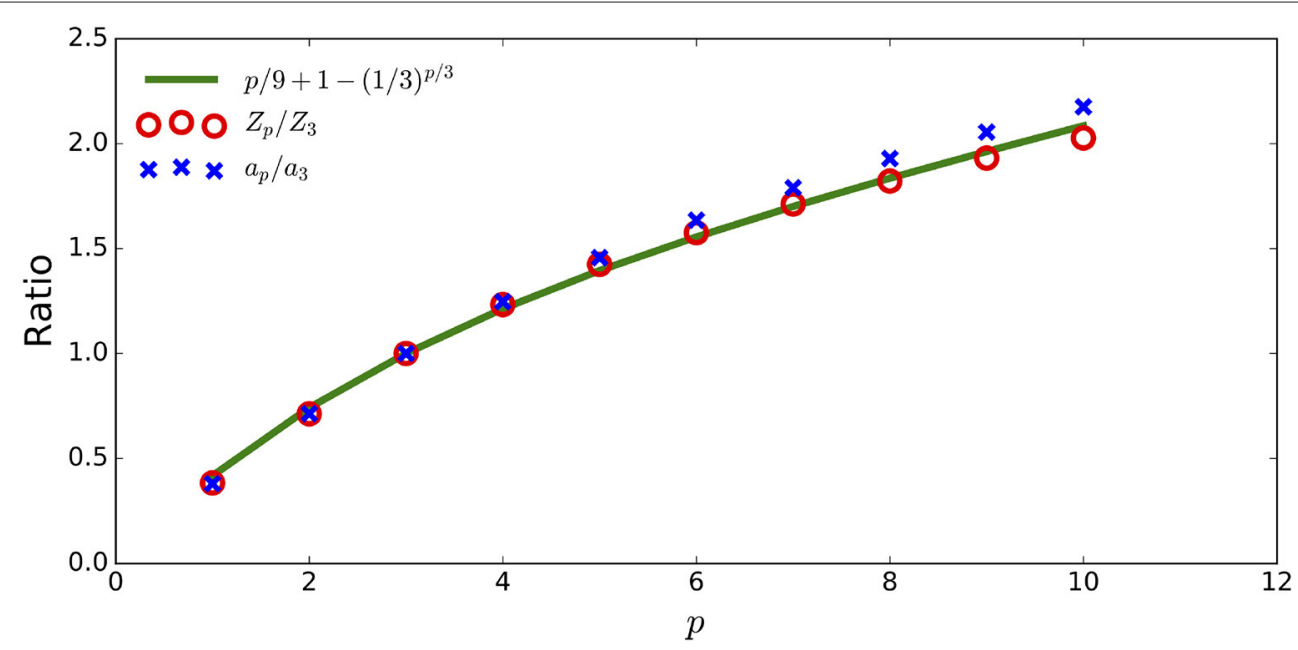

FIGURE 14 | The new ratio model agrees with the ratios of $Z_{p} / Z_{3}$ and of $a_{p} / a_{3}$ from JHTDB data in the dissipation range.

\subsection{Full $\zeta_{p}$ Parameterization}

We have developed a model that captures the longitudinal velocity increments for all of the small length scales up to and including the inertial range. The $\zeta_{p}$ parameterization starts with $\zeta_{p}=a_{p}$ at the Kolmogorov length scale $(l / \eta=1)$ as shown in Equation (17). Based on Equation (6.1), a model for $\zeta_{p}$ that captures the linear segment in the dissipative range for each $p$ with input $Z_{3}$ and $a_{3}$ value dependence is defined as:

$$
\begin{aligned}
\zeta_{p}^{d r} & =Z_{p} \cdot \ln \left(\frac{l}{\eta}\right)+a_{p} \\
& =\left(p / 9+1-(1 / 3)^{\frac{p}{3}}\right) \cdot\left(Z_{3} \cdot \ln \left(\frac{l}{\eta}\right)+a_{3}\right) .
\end{aligned}
$$

We have parameterized $\zeta_{p}$ for longitudinal velocity increments $\left\langle\left|\delta_{l} u\right|^{p}\right\rangle$ at the dissipation range with a finite number of parameters for $p$ up to 10 . It is $p$ dependent, with the variables $Z_{3}$ and $a_{3}$ calculated from $\zeta_{3}$.

The dissipation range begins at the Kolmogorov length scale and ends at the intersection with the theoretical value from SL, which marks the start of the inertial range. This transition occurs approximately at the Taylor microscale [19]. The transition point can be found by

$$
\begin{aligned}
\left(p / 9+1-(1 / 3)^{\frac{p}{3}}\right) & \cdot\left(Z_{3} \cdot \ln \left(\frac{l}{\eta}\right)+a_{3}\right)=\zeta_{p}^{d r} \\
& =\zeta_{p}^{S L}=p / 9+2 \cdot\left[1-(2 / 3)^{\frac{p}{3}}\right] .
\end{aligned}
$$

We solve this equation for length scale $l$ with the known variable $Z_{3}$ and $a_{3}$ from $\zeta_{3}$ data along with the known $\eta$. The $p$ dependent 
transition points of $\zeta_{p}$ for $p=2,3,6$ are shown as the thick plus symbols in Figure 12.

\section{THE SL CONJECTURE FOR THE LAMINAR LIMIT}

SL discusses the large $p$ asymptotes of $\epsilon_{l}^{p}$ in developing parameters for their $\tau_{p}$ inertial range methodology. They predicted dominance by vortices in this regime. We come to the same conclusion in the large $p$ asymptotes more directly through an analysis of $\zeta_{p}$, which reflects the intensity of the vortical structures. We find in Figure 13 and Equation (24) that $\zeta_{p}$ is increasing in $p$ as the length $l / \eta$ moves toward the dissipation range.

We interpret the length scale $l / \eta$ limit in terms of TaylorGreen vortices continued past the instability point. Assuming the CC model remains applicable in this range, Figure 11 analyzes the $C C_{\infty}$ model and shows a non-zero residual value for $T_{2}$ and similarly for all $T_{p}$. Line vortices do not dissipate energy and are described by the vanishing $\tau_{p}$ for all $p$. The presence of non-zero $T_{p}$ for all $p$ indicates that the line vortices occur in an unstable state, as occurs in a Taylor-Green vortex, continued past its singular value.

\section{CONCLUSIONS}

We have extended scaling laws for longitudinal velocity increments and energy dissipation rate structure functions from the inertial range to all length scales by modifying their exponential scaling exponents. We verify the complete parameterization models for $\tau_{p}$ and $\zeta_{p}$ in the dissipation range, i.e., from the Kolmogorov scale to the Taylor scale, limited to $p \leq 10$ for $\zeta_{p}$. Our major model feature, linearity of the log scale dissipation processes, is verified through comparison to the JHTDB and UMA data.

\section{REFERENCES}

1. Hinze JO. Turbulence. New York, NY: McGraw-Hill (1975).

2. Miller LP, Dimotakis EP. Stochastic geometric properties of scalar interfaces in turbulent jets. Phys Fluids A Fluid Dyn. (1991) 3:168-77.

3. She ZS, Leveque E. Universal scaling laws in fully developed turbulence. Phys Rev Lett. (1994) 72:336-9.

4. Kolmogorov AN. Local structure of turbulence in incompressible viscous fluid for very large Reynolds number. Doklady Akad Nauk SSSR. (1941) 30:299-3031

5. Frisch U. Turbulence: The Legacy of A. N. Kolmogorov. Cambridge: Cambridge Univeristy Press (1996).

6. Kolmogorov AN. A refinement of previous hypotheses concerning the local structure of turbulence in a viscous incompressible fluid at high Reynolds number. J Fluid Mech. (1962) 1 3:82-5.

7. Novikov EA. Infinitely divisible distributions in turbulence. Phys Rev E. (1994) 50:R3303-5.

8. Chen S, Cao N. Inertial range scaling in turbulence. Phys Rev E. (1995) 72:R5757-9.

9. Frick P, Dubrulle B, Babiano A. Scaling properties of a class of shell models. Phys Rev E. (1995) 51:5582-93.
In local regions, even within a fully developed turbulent flow, the turbulence is not isotropic nor scale invariant due to the influence of larger turbulent structures (or their absence). For this reason, turbulence that is not fully developed is an important issue which the present analysis addresses.

In Kolmogorov theory and in advanced multifractal scaling law theories, $\zeta_{2}$ has a range in which it is a flat line of constant value in log length scale, as observed. It has been noted that $\tau_{p}$ is not constant in the inertial range that is defined by $\zeta_{2}$. Our data analysis and data of others show no clearly defined inertial range for the energy dissipation rate. The $\tau_{p}$ peak is $p$ dependent, but occurs approximately at the Taylor micro-scale. The transition from the dissipation range to the inertial range takes place near the Taylor micro-scale.

We find that the Chen and Cao model for $\tau_{p}$ can be extended across all length scales for small $p$ moments. Our refined $C C_{q}$ model describes the relation between any $\tau_{p}$ and $\tau_{q}$ exponents. The $C C_{\infty}$ model complements the SL analysis of vortices in the $l / \eta \rightarrow 1$ limit are given.

\section{DATA AVAILABILITY STATEMENT}

Publicly available datasets were analyzed in this study. This data can be found here: http://turbulence.pha.jhu.edu/.

\section{AUTHOR CONTRIBUTIONS}

$\mathrm{AH}$ took the lead in the numerical analysis of the DNS data and in some of the discovery of the analysis algorithms. RK took the lead in designing the numerical algorithms had a major role in the interpolation of the data. JG lead in the overall planning and formulation of the study outline. All authors contributed to all aspects of the study, numerics, analysis, and conceptual modeling.

10. Boldyrev S, Nordlund A, Padoan P. Scaling relations of supersonic turbulence in star-forming molecular clouds. Astrophys J. (2002) 573:678-84. doi: $10.1086 / 340758$

11. Müller WC, Biskamp D. Scaling properties of three-dimensional magnetohydrodynamic turbulence. Phys Rev Lett. (2000) 84:475-8. doi: 10.1103/PhysRevLett.84.475

12. Chavarria G, Baudet $C$, Benzi R, Ciliberto S. Hierarchy of the velocity structure functions in fully developed turbulence. J Phys. (1995) 5:485-90.

13. Chavarria GR, Baudet C, Ciliberto S. Hierarchy of the energy dissipation moments in fully developed turbulence. Phys Rev Lett. (1995) 74:1986-9.

14. Chavarria G, Baudet C, Benzi R, Ciliberto S. Scaling laws and dissipation scale of a passive scalar in fully developed turbulence. Phys D Nonlinear Phenomena. (1996)99:369-80.

15. Zou Z, Zhu Y, Zhou M, She ZS. Hierarchical structures in a turbulent pipe flow. Fluid Dyn Res. (2003) 33:493-508. doi: 10.1016/j.fluiddyn.200 3.07.002

16. Cao N, Chen S, She ZS. Scalings and relative scalings in the Navier-Stokes turbulence. Phys Rev Lett. (1996) 76:3711-4.

17. Chevillard L, Castaing B, Leveque E, Arneodo A. Unified multifractal description of velocity increments statistics in turbulence: intermittency and skewness. Phys D Nonlinear Phenomena. (2006) 218:77-82. doi: 10.1016/j.physd.2006.04.011 
18. Lundgren TS. Linearly Forced Isotropic Turbulence. Center for Turbulence Research Annual (2003).

19. Obligado M, Vassilicos J. The non-equilibrium part of the inertial range in decaying homogeneous turbulence. Europhys Lett. (2019) 127:64004. doi: 10.1209/0295-5075/127/ 64004

20. Meldi M, Sagaut P. Turbulence in a box: quantification of large-scale resolution effects in isotropic turbulence free decay. J Fluid Mech. (2017) 818:697-715. doi: 10.1017/jfm.201 7.158

21. Li Y, Perlman E, Wan M, Yang Y, Burns R, Meneveau C, et al. A public turbulence database cluster and applications to study Lagrangian evolution of velocity increments in turbulence. J Turbulence. (2008) 9:1-29. doi: 10.1080/146852408023 76389

22. Perlman E, Burns R, Li Y, Meneveau C. Data exploration of turbulence simulations using a database cluster. In: SC '07 Proceedings of the 2007 ACM/IEEE Conference on Supercomputing. Seattle, WA (2007).

23. He G, Doolen GD, Chen S. Calculations of longitudinal and transverse velocity structure functions using a vortex model of isotropic turbulence. Phys Fluids. (1999) 11:3743-8.
24. Almalkie S, De Bruyn Kops S. Energy dissipation rate surrogates in incompressible Navier-Stokes turbulence. J Fluid Mech. (2012) 697:204-36. doi: $10.1017 /$ jfm.2012.53

Conflict of Interest: The authors declare that the research was conducted in the absence of any commercial or financial relationships that could be construed as a potential conflict of interest.

Publisher's Note: All claims expressed in this article are solely those of the authors and do not necessarily represent those of their affiliated organizations, or those of the publisher, the editors and the reviewers. Any product that may be evaluated in this article, or claim that may be made by its manufacturer, is not guaranteed or endorsed by the publisher.

Copyright (c) $2022 \mathrm{Hsu}$, Kaufman and Glimm. This is an open-access article distributed under the terms of the Creative Commons Attribution License (CC BY). The use, distribution or reproduction in other forums is permitted, provided the original author(s) and the copyright owner(s) are credited and that the original publication in this journal is cited, in accordance with accepted academic practice. No use, distribution or reproduction is permitted which does not comply with these terms. 